\title{
An irregular narrow complex tachycardia: atrial fibrillation or something else?
}

\author{
M. J. Mulder · C. P. Allaart · H. A. Hauer - M. J. B. Kemme
}

Published online: 14 December 2018

(C) The Author(s) 2018

\section{Answer}

The intracardiac recording during tachycardia (Fig. 1) shows a regular atrial rhythm, excluding atrial fibrillation. His bundle activation precedes ventricular activation and there is atrioventricular dissociation. The main differential diagnosis comprises atrioventricular node reentrant tachycardia (AVNRT), which may occur without atrial activation, and focal junctional tachycardia (FJT). Distinction is possible by analysing the response to a sinus beat which occurs before junctional depolarisation when the His bundle is non-

Fig. 1 Tachycardia during electrophysiology study with corresponding ladder diagram. Solid dots represent ectopic foci from the atrioventricular junction. Note the lack of retrograde His-atrial conduction. The second sinus beat (asterisk) occurs prior to the anticipated junctional ectopic beat (open circle) and advances His bundle and ventricular activation without terminating or resetting the tachycardia. This response confirms the diagnosis of focal junctional tachycardia ( $A$ atrium, $A V N$ atrioventricular node, CSp proximal coronary sinus, $H$ His bundle, HISd distal His bundle, $\checkmark$ ventricle)

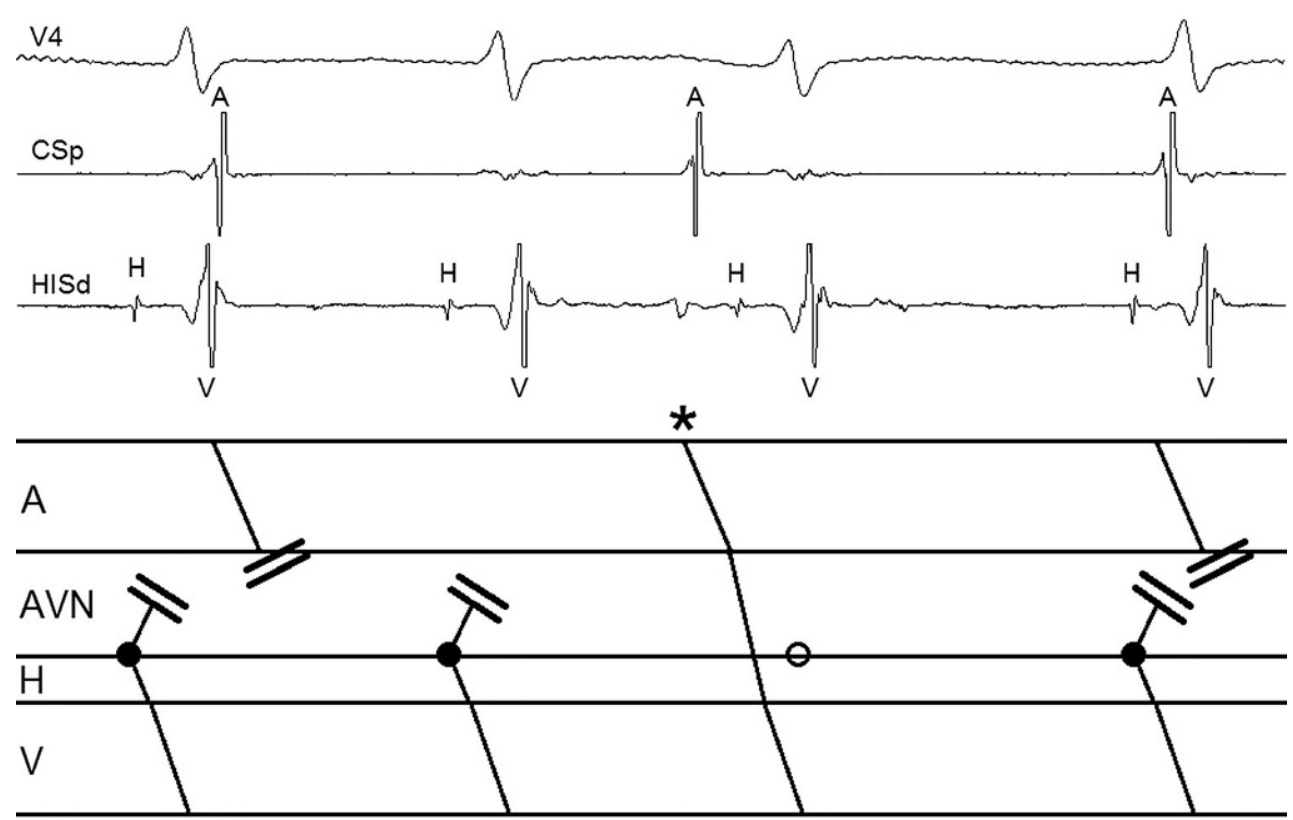

M. J. Mulder ( $₫)$ C. P. Allaart · H. A. Hauer · M. J. B. Kemme Department of Cardiology, Amsterdam UMC, Amsterdam Cardiovascular Sciences, Vrije Universiteit Amsterdam,

Amsterdam, The Netherlands

m.mulder2@vumc.nl

H. A. Hauer

Cardiology Centres of the Netherlands, Amsterdam, The

Netherlands 
refractory. Normal conduction of the sinus beat excludes AVNRT with bidirectional AH block. The sinus beat is conducted down the fast AV nodal pathway, leading to advancement of His bundle and ventricular activation without terminating or resetting the tachycardia. In common type (slow-fast) AVNRT, the subsequent refractoriness of the fast pathway would terminate the tachycardia. In contrast, termination is not expected during FJT, because of its focal nature [1]. Therefore, this tachycardia was diagnosed as FJT.

FJT is a rare arrhythmia in adults and is characterised by a rapid heart rate, narrow QRS complexes and atrioventricular dissociation. Frequently, an irregular rhythm is present which could lead to misdiagnosis of atrial fibrillation. The response to antiarrhythmic drugs is usually poor. Ablation is considered an effective alternative [2]. In this patient, after diagnosis of FJT, radiofrequency energy was delivered in the lower two-thirds of the Koch's triangle, during which the tachycardia converted to sinus rhythm. Atrioventricular conduction was preserved. She remained free of symptoms during 3 months of follow-up.

Conflict of interest M.J. Mulder, C.P. Allaart, H.A. Hauer and M.J.B. Kemme declare that they have no competing interests.
Open Access This article is distributed under the terms of the Creative Commons Attribution 4.0 International License (http://creativecommons.org/licenses/by/4.0/), which permits unrestricted use, distribution, and reproduction in any medium, provided you give appropriate credit to the original author(s) and the source, provide a link to the Creative Commons license, and indicate if changes were made.

\section{References}

1. Padanilam BJ, Manfredi JA, Steinberg LA, Olson JA, Fogel RI, Prystowsky EN. Differentiating junctional tachycardia and atrioventricular node re-entry tachycardia based on response to atrial extrastimulus pacing. J Am Coll Cardiol. 2008;52:1711-7.

2. Blomstrom-Lundqvist C, Scheinman MM, Aliot EM, et al. ACC/AHA/ESC guidelines for the management of patients with supraventricular arrhythmias-executive summary: a report of the American College of Cardiology/American Heart Association Task Force on Practice Guidelines and the European Society of Cardiology Committee for Practice Guidelines (Writing Committee to Develop Guidelines for the Management of Patients with Supraventricular Arrhythmias). Circulation. 2003;108:1871-909. 